\title{
Unsteady Electromagnetic Free Convection Micropolar Fluid Flow through a Porous Medium along a Vertical Porous Plate
}

\author{
Mohammad Rafiqul Islam ${ }^{1 *}$, Sonia Nasrin'², Md. Mahmud Alam ${ }^{3}$ \\ ${ }^{1}$ Department of Mathematics, Bangabandhu Sheikh Mujibur Rahman Science and Technology University, Gopalganj, Bangladesh \\ ${ }^{2}$ Department of Mathematics, Jagannath University, Dhaka, Bangladesh \\ ${ }^{3}$ Mathematics Discipline, Khulna University, Khulna, Bangladesh \\ Email: *mribsmrstu@yahoo.com
}

How to cite this paper: Islam, M.R., Nasrin, S. and Alam, M.M. (2020) Unsteady Electromagnetic Free Convection Micropolar Fluid Flow through a Porous Medium along a Vertical Porous Plate. Open Journal of Applied Sciences, 10, 701-718. https://doi.org/10.4236/ojapps.2020.1011049

Received: September 25, 2020 Accepted: November 17, 2020 Published: November 20, 2020

Copyright $\odot 2020$ by author(s) and Scientific Research Publishing Inc. This work is licensed under the Creative Commons Attribution International License (CC BY 4.0).

http://creativecommons.org/licenses/by/4.0/

\begin{abstract}
Unsteady electromagnetic free convection flows of two-dimensional micropolar fluid through in a porous medium parallel to a vertical porous plate have been investigated numerically. Similarity analysis has been used to transform the governing equations into its non-dimensional form by using the explicit finite difference method to obtain numerical solutions. Estimated results have been gained for various values of Prandtl number, Grashof number, material parameters, micropolar parameter, electric conductivity, electric permeability, thermal relaxation time and the permeability of the porous medium. The effects of pertinent parameters on the velocity, electric induction, magnetic induction, microrotation and temperature distributions have been investigated briefly and illustrated graphically.
\end{abstract}

\section{Keywords}

Micropolar Fluid, Free Convection, Porous Medium, Explicit Finite Difference

\section{Introduction}

Fluids with microstructure are micropolar fluids which are randomly oriented or composed of spherical particles that are rigid with their rotation and also ceased in a viscous medium. It has been known that Navier-Stokes equations are unable to explain the phenomena at micro and nanoscales; on the other hand, MFD can express the physical phenomena at micro and nanoscales owing to its additional degree of freedom for circulation. Physical examples of micropolar fluids may present in the non-Newtonian fluids, blood flows, polymer fluids and liquid 
crystals and all of them containing intrinsic polarities. The presence of dust or fumes in a gas can be especially modeled using micropolar fluid dynamics. The porous media heat transfer problems have various practical uses in engineering applications such as geothermal systems, crude oil extraction and groundwater pollution. Eringen first proposed [1] and [2] the general theory of micropolar fluids which illustrate certain microscopic effects arising from the microstructure and micro motions of the fluid flow. The interaction of natural convection with thermal radiation in laminar boundary layer flow over an isothermal, horizontal flat plate is studied by Ali et al. [3]. Harutha and Devasena [4] investigated the steady mixed convection flow of a viscous incompressible micropolar fluid through a porous medium towards a stagnation point over a vertical surface when the buoyancy forces assist. Hudimoto and Tokuoka [5] have devised the two-dimensional parallel shear flow of a linear micropolar fluid. They analyzed and compared it with the colloidal suspensions. Rees and Pop [6] expressed the steady micropolar free convection fluid flow from a vertical isothermal flat plate. Elbarbary [7] discussed a new Chebyshev finite difference method is proposed for solving the governing equations of the boundary layer flow. Nandhini and Ramya [8] analyzed the heat and mass transfer of the free convection flow in a micropolar fluid past an inclined stretching sheet. Hassanien and Glora [9] analyzed the heat transfer on a non-isothermal stretching sheet to a micropolar fluid. Kartini Ahmad et al. [10] described a micropolar fluid flow and heat transfer past a non-linearly stretching plate. Khonsari and Brewe [11] investigated and compared the parameters of micropolar fluids with finite length lubricated that resulted significantly higher load carrying capacity than Newtonian fluids. Effects of free convection currents with one relaxation time on the flow of a viscoelastic conduction fluid through a porous medium, which is bounded by a vertical plane surface, have studied by Ezzatand Abd-Ellal [12]. Edlabe and Mohammed [13] determined the heat and mass transfer occurring in the hydromagnetic flow of the non-Newtonian fluid on a linearly accelerating surface with temperature dependent heat source subject to suction or blowing. Edlabe and Ouaf [14] are obtained the heat and mass transfer in a hydro magnetic flow of a micropolar fluid past a stretching surface with Ohmic heating and viscous dissipation. Aydin and Pop [15] analyzed the two-dimensional steady laminar natural convective flow and heat transfer of micropolar fluids in a square enclosure. Muthu et al. [16] investigated the oscillatory flow of micropolar fluid in an annular region with constriction, provided by variation of the outer tube radius. Glora [17] presented an unsteady combined convection of a micropolar fluid among a vertical plate. Hsu and Wang [18] presented a numerical study of the laminar mixed convection of micropolar fluids in a square cavity with localized heat source Lok et al. [19] studied a microplar mixed convection boundary layer fluid flow near the region of the stagnation point of on a double-infinite vertical flat plate. Zakaria [20] investigated the influence of a transverse magnetic field on the motion of an electrically conducting micropolar 
fluid through a porous medium in one-dimensional and used the Laplace transformation with $\varepsilon$-algorithm technique to find its solution in the Laplace transformation domain numerically.

In the present work, our aim is to study that the numerical investigation on unsteady two-dimensional electromagnetic free convection micropolar fluid flows through a porous medium along a vertical porous plate. The obtained equations are non-linear coupled partial differential equations, which are solved by using explicit finite difference method and the results are shown graphically and also discussed its behavior in detail for the velocity, induced magnetic field, induced electric field, micro rotation and temperature distribution with respect to its pertinent parameters.

\section{Problem Formulation}

Considered unsteady MHD micropolar fluid flow embedded in a porous medium along a vertical porous plate. The velocity at the wall is zero and also outside of the boundary layer is zero. The temperature of the plate is raised from $T_{w}$ to $T_{\infty}$, where $T_{w}$ and $T_{\infty}$ is the temperature at the plate and outside of the boundary layer respectively. The magnetic Reynolds number is taken large enough so that the induced magnetic field equation is considerable for our assumption. The Physical model of the system is shown in the following Figure 1.

The flow is governed by the equation of continuity, the momentum equation, induction magnetic field equation, the electric field equation, angular momentum equation and the energy equation are as follows:

$$
\frac{\partial u^{+}}{\partial x^{+}}+\frac{\partial v^{+}}{\partial y}=0
$$

$$
\begin{aligned}
& \frac{\partial u^{+}}{\partial t^{+}}+u^{+} \frac{\partial u^{+}}{\partial x^{+}}+v^{+} \frac{\partial u^{+}}{\partial y} \\
& =g \beta\left(T^{+}-T_{\infty}^{+}\right)+\left(\frac{\mu+\mu^{*}}{\rho}\right) \frac{\partial^{2} u^{+}}{\partial y^{+2}}+\frac{\mu^{*}}{\rho} \frac{\partial N^{+}}{\partial y^{+}}+\frac{\alpha^{2}}{H_{0}}\left(\frac{\partial H_{x}^{+}}{\partial y^{+}}+\varepsilon_{0}^{+} \frac{\partial E^{+}}{\partial t^{+}}\right)-\frac{\mu}{\rho \kappa} u^{+}
\end{aligned}
$$

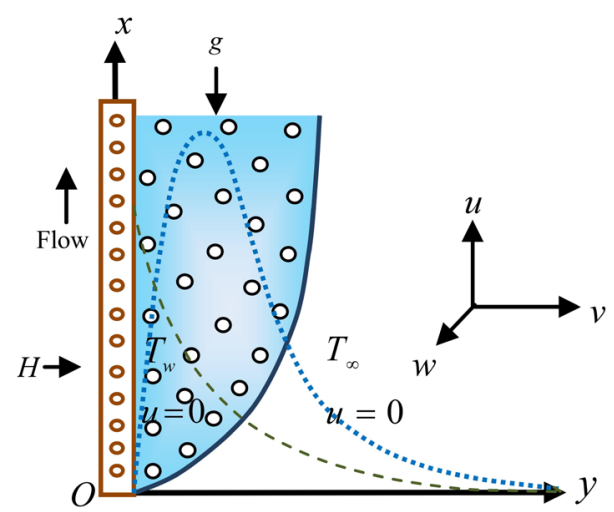

Figure 1. Physical Model of the system. 


$$
\begin{gathered}
\frac{\partial H_{x}^{+}}{\partial t^{+}}+u^{+} \frac{\partial H_{x}^{+}}{\partial x^{+}}+v^{+} \frac{\partial H_{x}^{+}}{\partial y^{+}}=v_{m} \frac{\partial^{2} H_{x}^{+}}{\partial y^{+2}}-\frac{\varepsilon_{0}^{+}}{\sigma_{0}^{+}} \frac{\partial^{2} H_{x}^{+}}{\partial t^{+2}}+H_{0} \frac{\partial u^{+}}{\partial y^{+}} \\
\frac{\partial E^{+}}{\partial t^{+}}+u^{+} \frac{\partial E^{+}}{\partial x^{+}}+v^{+} \frac{\partial E^{+}}{\partial y^{+}}=-\frac{\sigma_{0}^{+}}{\varepsilon_{0}^{+}} E^{+}-\frac{1}{\varepsilon_{0}^{+}} \frac{\partial H_{x}^{+}}{\partial y^{+}}-\frac{H_{0} \sigma_{0}^{+} \mu_{0}}{\varepsilon_{0}^{+}} u^{+} \\
\frac{\partial N^{+}}{\partial t^{+}}+u^{+} \frac{\partial N^{+}}{\partial x^{+}}+v^{+} \frac{\partial N^{+}}{\partial y^{+}}=\frac{\gamma}{\rho j} \frac{\partial^{2} N^{+}}{\partial y^{+2}}-\frac{\mu^{*}}{\rho j}\left(\frac{\partial u^{+}}{\partial y^{+}}+2 N^{+}\right) \\
\frac{\partial T^{+}}{\partial t^{+}}+u^{+} \frac{\partial T^{+}}{\partial x^{+}}+v^{+} \frac{\partial T^{+}}{\partial y^{+}}=\frac{k}{\rho c_{p}} \frac{\partial^{2} T^{+}}{\partial y^{+2}}-\tau_{0}^{+} \frac{\partial^{2} T^{+}}{\partial t^{+2}}
\end{gathered}
$$

with boundary conditions are as follows:

$$
\begin{aligned}
& y^{+}=0: \quad u^{+}=0, \quad N^{+}=0, \quad T^{+}=T_{w}^{+}, \quad H_{x}^{+}=0 \\
& y^{+} \rightarrow \infty: \quad u^{+} \rightarrow 0, \quad N^{+} \rightarrow 0, T^{+} \rightarrow T_{\infty}^{+}, H_{x}^{+} \rightarrow 0
\end{aligned}
$$

\section{Similarity Analysis}

Now introducing the following non-dimensional quantities as

$$
\begin{aligned}
& \quad x=\frac{\alpha \rho}{\mu} x^{+}, y=\frac{\alpha \rho}{\mu} y^{+}, u=\frac{u^{+}}{\alpha}, w=\frac{w^{+}}{\alpha}, H=\frac{H_{x}^{+}}{H_{0}}, E=\frac{E^{+}}{\mu_{0} H_{0} \alpha}, \\
& t=\frac{\alpha^{2} \rho}{\mu} t^{+}, \theta=\frac{T^{+}-T_{\infty}^{+}}{T_{w}^{+}-T_{\infty}^{+}}, N=\frac{\mu}{\alpha^{2} \rho} N^{+}, \varepsilon_{0}=\mu_{0} \alpha^{2} \varepsilon_{0}^{+}, \sigma_{0}=\frac{\mu \mu_{0}}{\rho} \sigma_{0}^{+} \\
& \text {and } \tau_{0}=\frac{\alpha^{2} \rho}{\mu} \tau_{0}^{+} .
\end{aligned}
$$

Using these quantities into the above Equations (1)-(7), we obtain the following dimensionless form of the given equations:

$$
\begin{gathered}
\frac{\partial u}{\partial x}+\frac{\partial v}{\partial y}=0 \\
\frac{\partial u}{\partial t}+u \frac{\partial u}{\partial x}+v \frac{\partial u}{\partial y}=G_{r} \theta+(1+R) \frac{\partial^{2} u}{\partial y^{2}}+R \frac{\partial N}{\partial y}+\frac{\partial H}{\partial y}+\varepsilon_{0} \frac{\partial E}{\partial t}-\frac{u}{K} \\
\frac{\partial H}{\partial t}+u \frac{\partial H}{\partial x}+v \frac{\partial H}{\partial y}=\frac{1}{b} \frac{\partial^{2} H}{\partial y^{2}}-\frac{\varepsilon_{1}}{b} \frac{\partial^{2} H}{\partial t^{2}}+\frac{\partial u}{\partial y} \\
\frac{\partial E}{\partial t}+u \frac{\partial E}{\partial x}+v \frac{\partial E}{\partial y}=-\frac{\sigma_{0}}{\varepsilon_{0}} E-\frac{\sigma_{0}}{\varepsilon_{0}} u-\frac{1}{\varepsilon_{0}} \frac{\partial H}{\partial y} \\
\frac{\partial N}{\partial t}+u \frac{\partial N}{\partial x}+v \frac{\partial N}{\partial y}=\lambda \frac{\partial^{2} N}{\partial y^{2}}-\sigma\left(\frac{\partial u}{\partial y}+2 N\right) \\
\frac{\partial \theta}{\partial t}+u \frac{\partial \theta}{\partial x}+v \frac{\partial \theta}{\partial y}=\frac{1}{P_{r}} \frac{\partial^{2} \theta}{\partial y^{2}}-\tau_{0} \frac{\partial^{2} \theta}{\partial t^{2}}
\end{gathered}
$$

with the corresponding boundary conditions:

$$
\begin{aligned}
& y=0: u=0, \quad N=0, \quad \theta=1, \quad H=0 \\
& y \rightarrow \infty: u \rightarrow 0, \quad N \rightarrow 0, \quad \theta \rightarrow 0, \quad H \rightarrow 0
\end{aligned}
$$


where $G_{r}=\beta g \mu\left(T_{w}^{+}-T_{\infty}^{+}\right) /\left(\rho \alpha^{3}\right)$ is the Grashof number, $P_{r}=c_{p} \mu / k$ is the Prandtl number, $R=\mu^{*} / \mu$ is the micropolar parameter, $K=\rho^{2} \alpha^{2} \kappa / \mu^{2}$ is the Permeability parameter. Also $\sigma=\mu \mu^{*} /\left(\alpha^{2} j \rho^{2}\right), \quad b=\mu / \rho v_{m}, \varepsilon_{1}=b \varepsilon_{0} / \sigma_{0}$ and $\lambda=\gamma / j \mu$ are the dimensionless material parameter.

\section{Method of Solution}

The explicit finite difference method has been used to solve the governing non-linear coupled dimensionless partial differential Equations (8) to (13) together with its boundary conditions. The finite difference schemes with respect to $t, x$ and $y$ are as follows:

$$
\begin{gathered}
\frac{\partial u}{\partial t}=\frac{U_{i, j}^{k+1}-U_{i, j}^{k}}{\Delta t} ; \frac{\partial u}{\partial x}=\frac{U_{i, j}^{k}-U_{i-1, j}^{k}}{\Delta x} ; \\
\frac{\partial u}{\partial y}=\frac{U_{i, j}^{k}-U_{i, j-1}^{k}}{\Delta y} ; \frac{\partial^{2} H}{\partial t^{2}}=\frac{h_{i, j}^{k+2}-2 h_{i, j}^{k+1}+h_{i, j}^{k}}{\Delta t^{2}} ; \\
\frac{\partial^{2} u}{\partial y^{2}}=\frac{U_{i, j+1}^{k}-2 U_{i, j}^{k}+U_{i, j-1}^{k}}{\Delta y^{2}}
\end{gathered}
$$

Here, the subscript $i$ and $j$ refer to $x$ and $y$ and the superscript $k$ refers to time $t$. Finite difference Schemes for the other variables have been written in the same way. The graphical representations of this problem have been illustrated by using Compaq visual FORTRAN 6.6 a tools.

\section{Results and Discussion}

The behavior of the velocity $(u)$, induced magnetic field $(H)$, induced electric field $(E)$, microrotation $(N)$ and temperature $(\theta)$ distributions have been analyzed for the different values of Prandtl number $\left(P_{r}\right)$, Grashof number $\left(G_{r}\right)$, permeability of porous medium $(K)$, micropolar parameter $(R)$ and thermal relaxation time $\left(\tau_{0}\right)$ with the values of time $t=1$. The flow characteristics have been shown graphically from Figures 2-25.

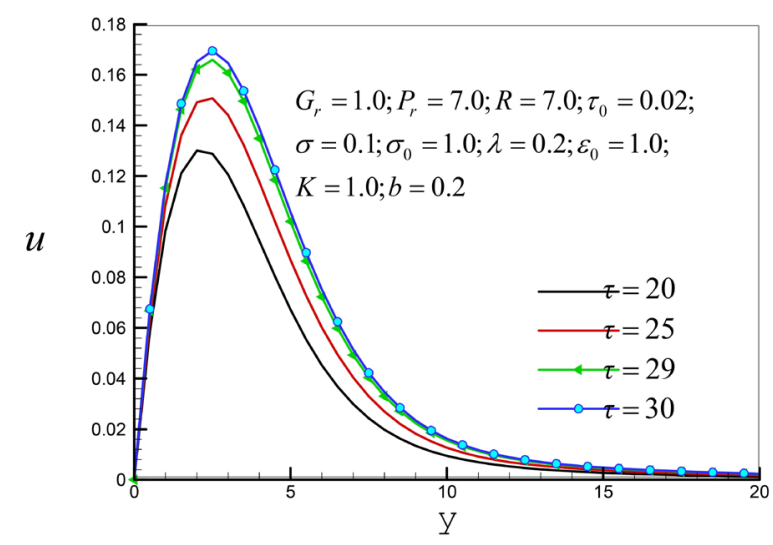

Figure 2. Time sensitivity on velocity $u$. 


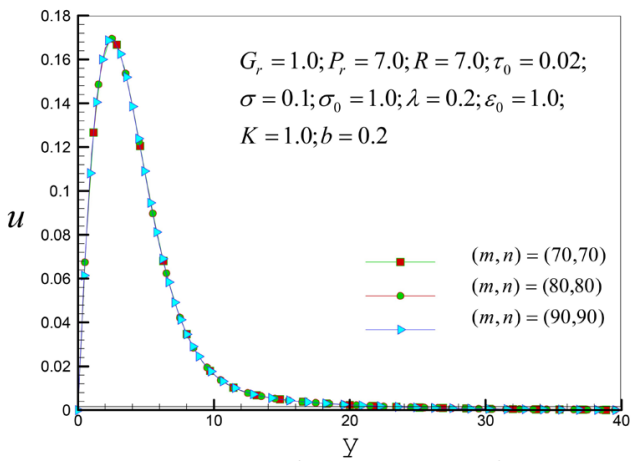

Figure 3. Mesh sensitivity on velocity $u$.

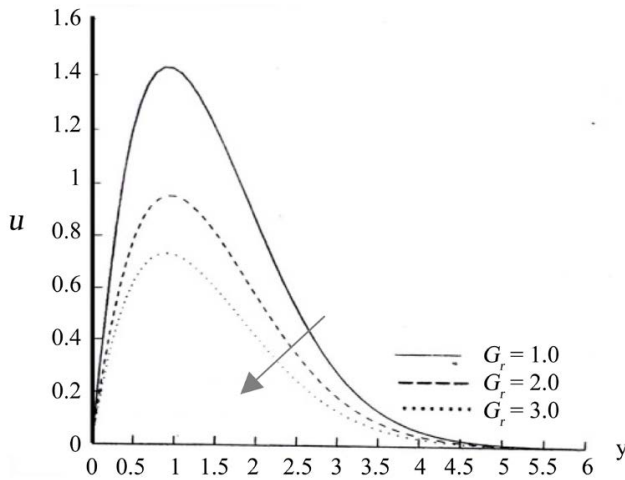

(a)

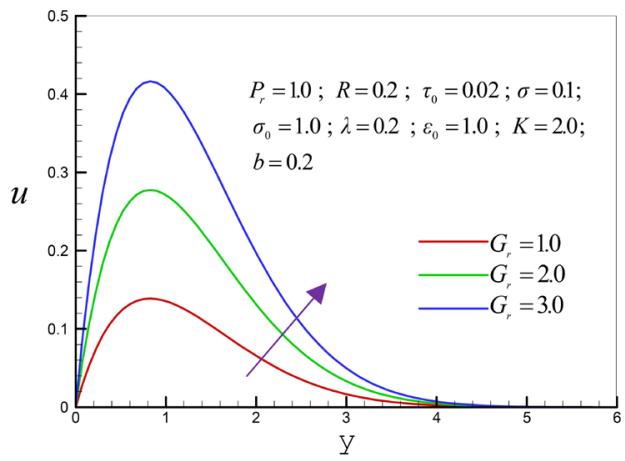

(b)

Figure 4. (a) Velocity distribution $u$ for different values of $G_{r}$ [M. Zakaria [20]; (b) Velocity distribution $u$ for different values of $G_{r}$ [Our Results].

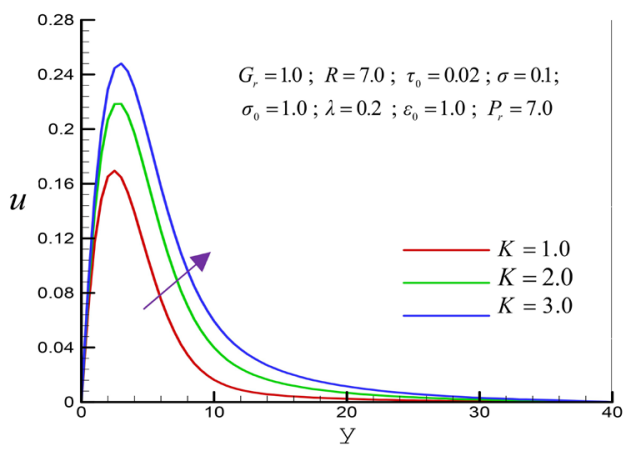

Figure 5. Velocity distribution $u$ for different values of $K$. 


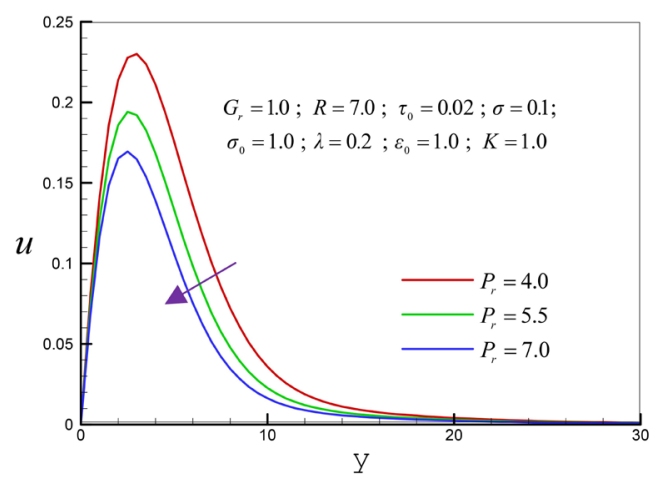

Figure 6. Velocity distribution $u$ for different values of $P_{r}$

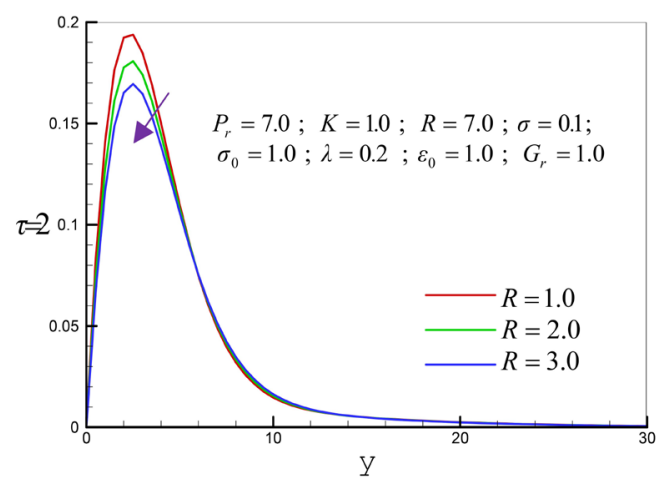

Figure 7. Velocity distribution $K$ for different values of $R$.

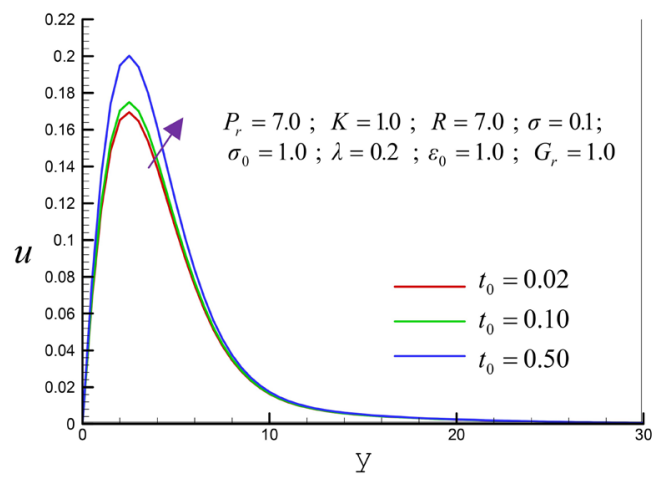

Figure 8. Velocity distribution $u$ for different values of $t_{0}$.

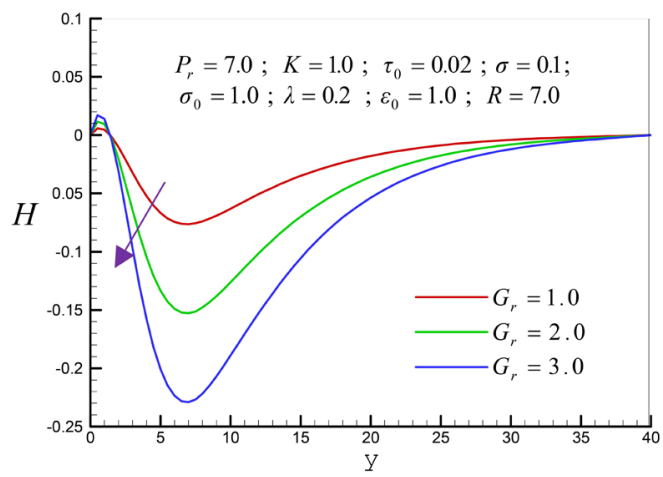

Figure 9. Magnetic field distribution $H$ for different values of $G_{r}$. 


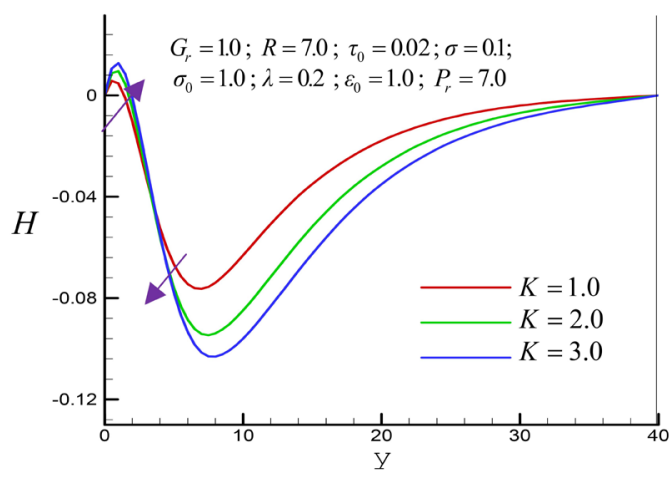

Figure 10. Induced Magnetic field distribution $H$ for different values of $K$.

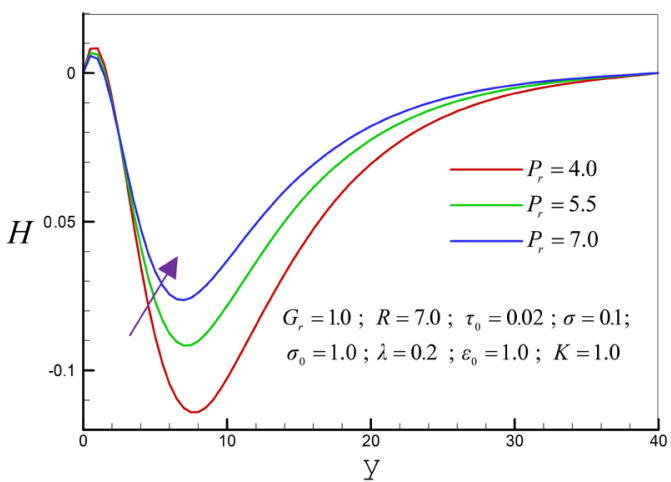

Figure 11. Induced Magnetic field distribution $R$ for different values of $P_{r}$.

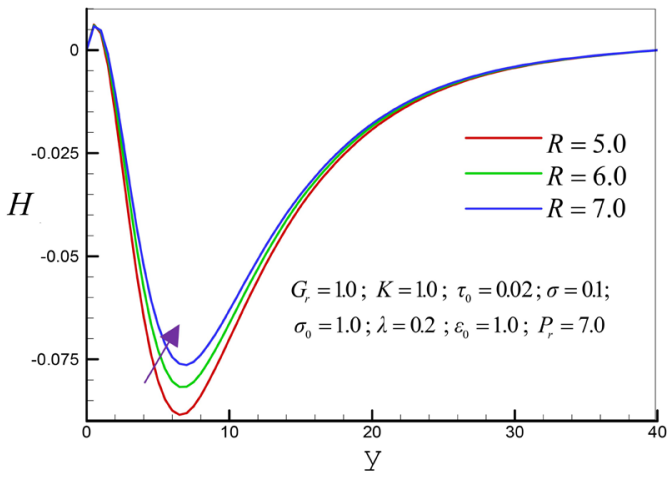

Figure 12. Induced Magnetic field distribution $H$ for different values of $R$.

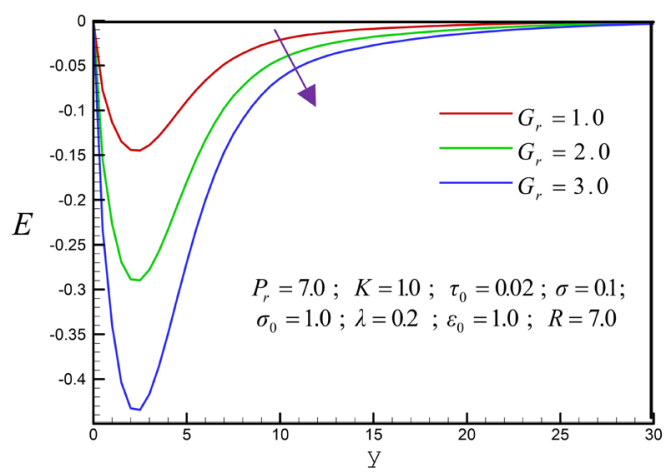

Figure 13. Induced electric field distribution $K$ for different values of $G_{r}$. 


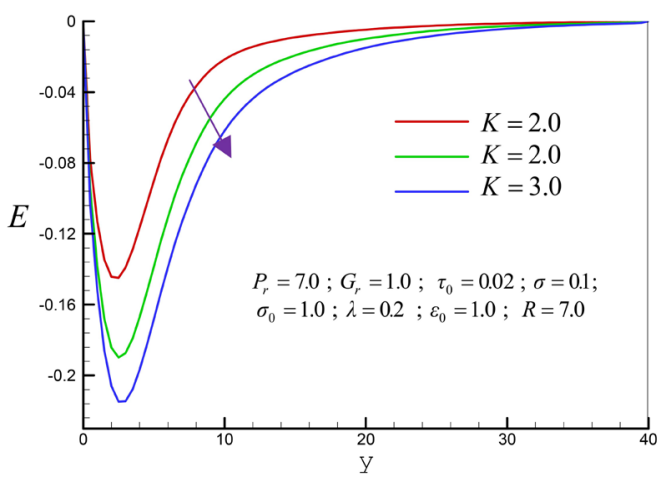

Figure 14. Induced electric field distribution $E$ for different values of $K$.

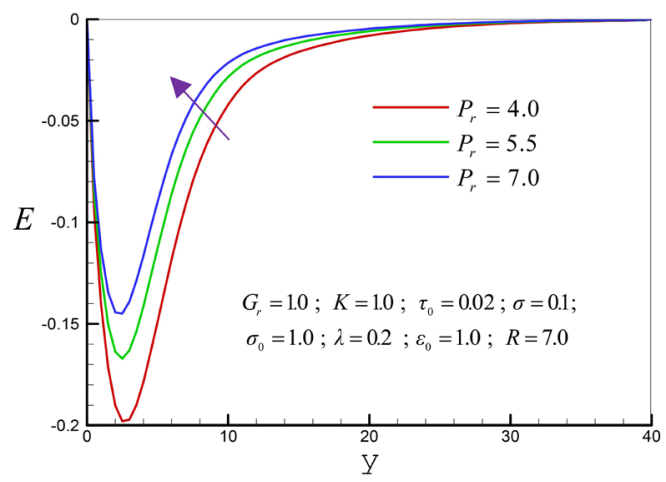

Figure 15. Induced electric field distribution $E$ for different values of $P_{r}$

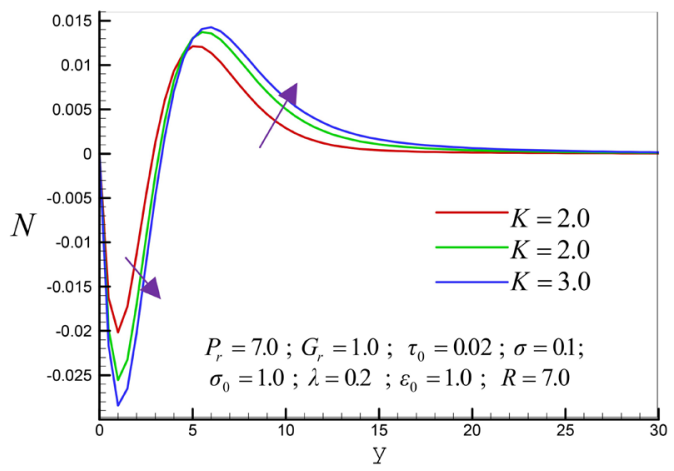

Figure 16. Microrotation distribution $N$ for different values of $K$.

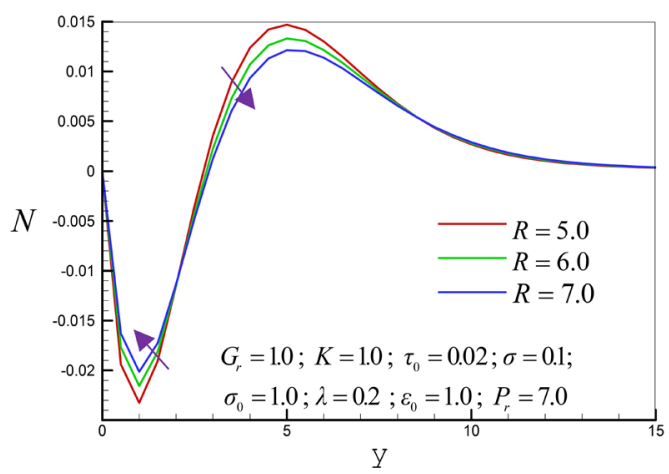

Figure 17. Microrotation distribution $N$ for different values of $R$. 


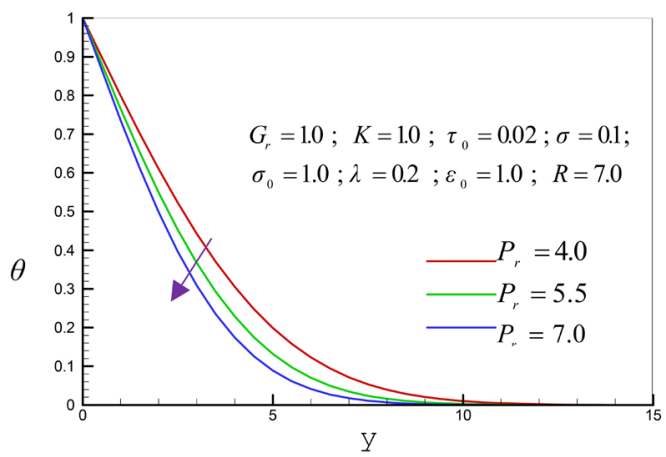

Figure 18. Temperature distribution $\theta$ for different values of $P_{r}$.

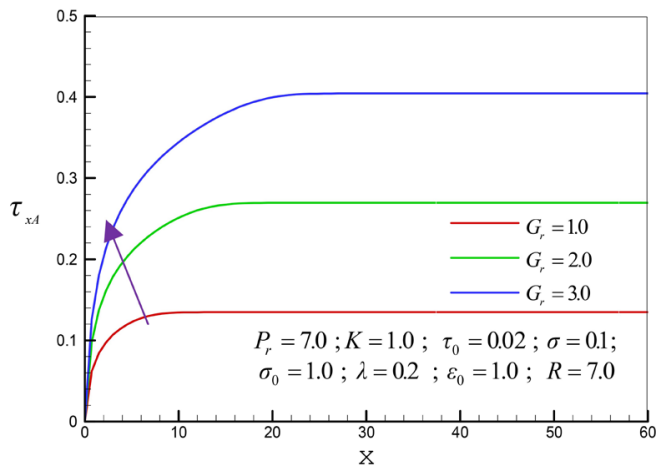

(a)

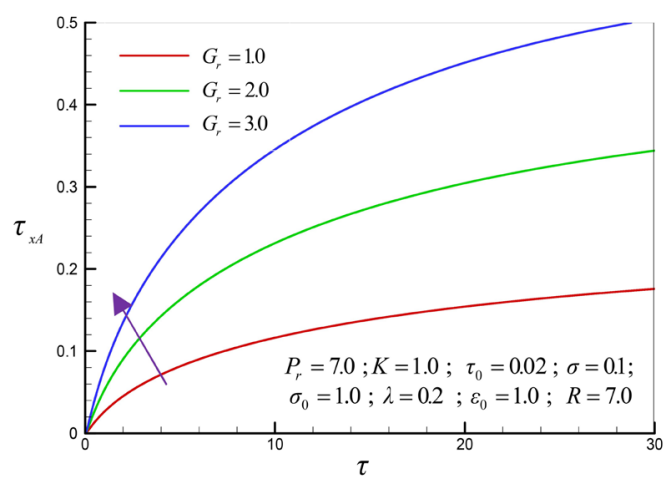

(b)

Figure 19. (a) Illustration of local shear stress $\tau_{x L}$ for different values of $G_{r}$ (b) Illustration of Average shear Stress $\tau_{x A}$ for different values of $G_{r}$.

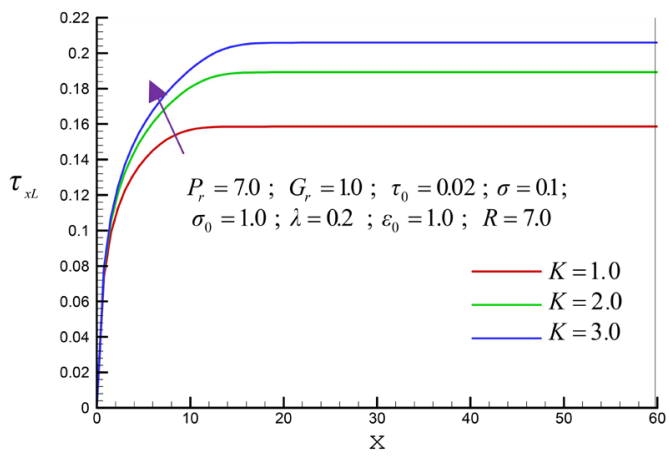

(a) 


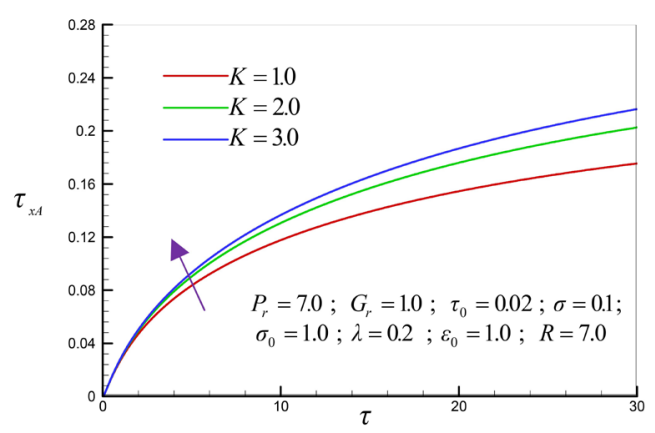

(b)

Figure 20. (a) Illustration of local shear Stress $\tau_{x L}$ for different values of $K$; (b). Illustration of average shear Stress $\tau_{x A}$ for different values of $K$.

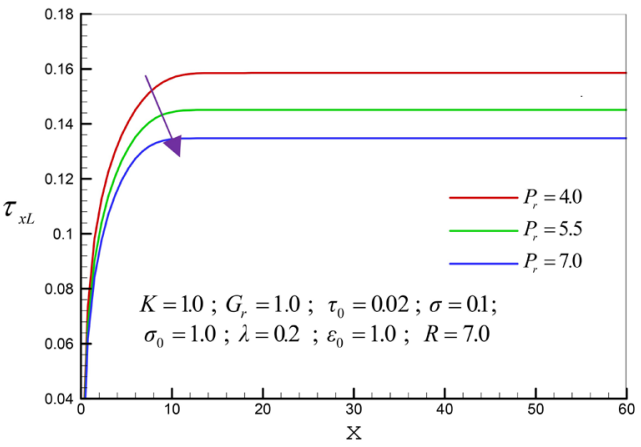

(a)

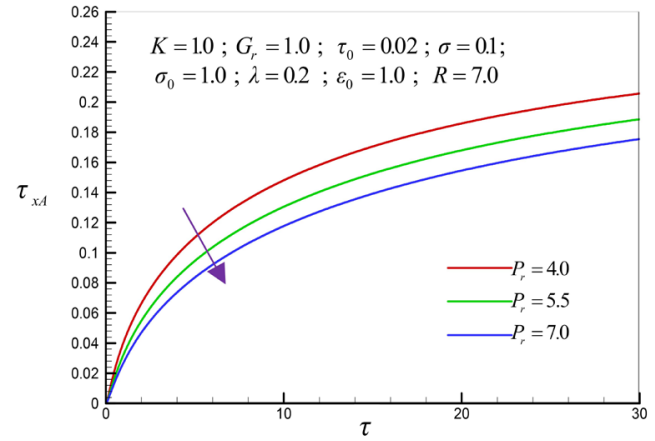

(b)

Figure 21. (a) Illustration of local shear stress $\tau_{x L}$ for different values of $P_{r}$; (b). Illustration of local average shear stress $\tau_{x A}$ for different values of $P_{r}$.

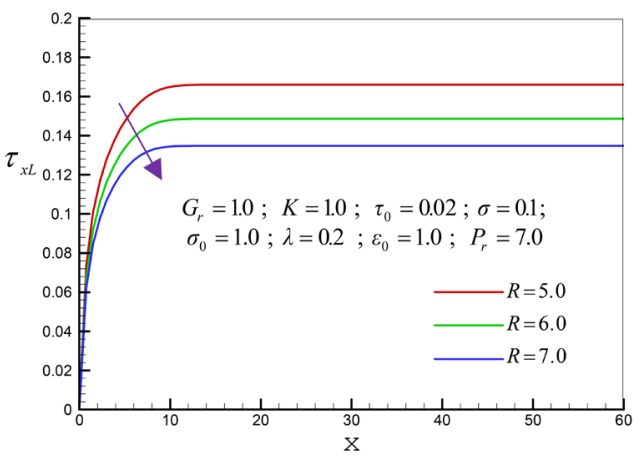

(a) 


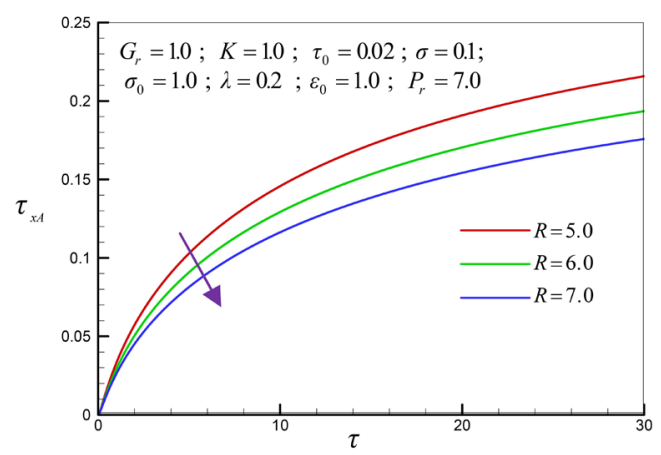

(b)

Figure 22. (a) Illustration of local shear stress $\tau_{x L}$ for different values of $R$; (b). Illustration of average shear stress $\tau_{x A}$ for different values of $R$.

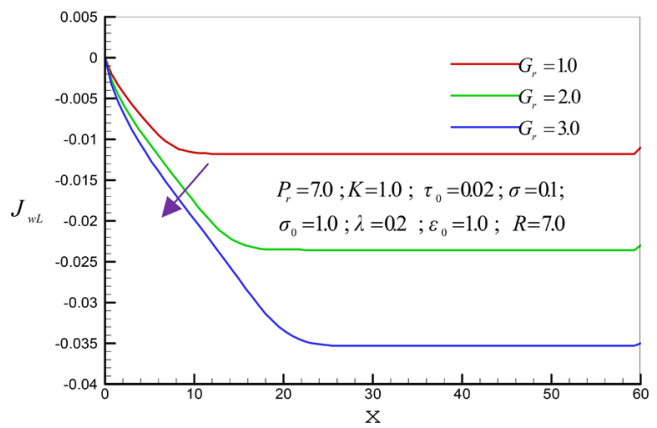

(a)

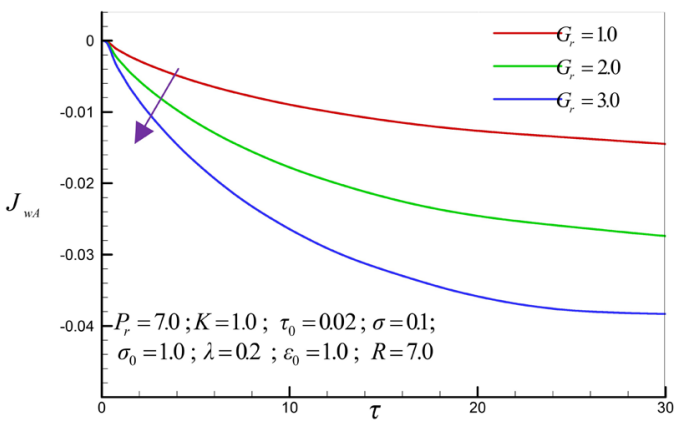

(b)

Figure 23. (a) Illustration of Current density $J_{w L}$ for different values of $G_{r}$; (b). Illustration of average Current Density $J_{w A}$ for different values of $G_{r}$.

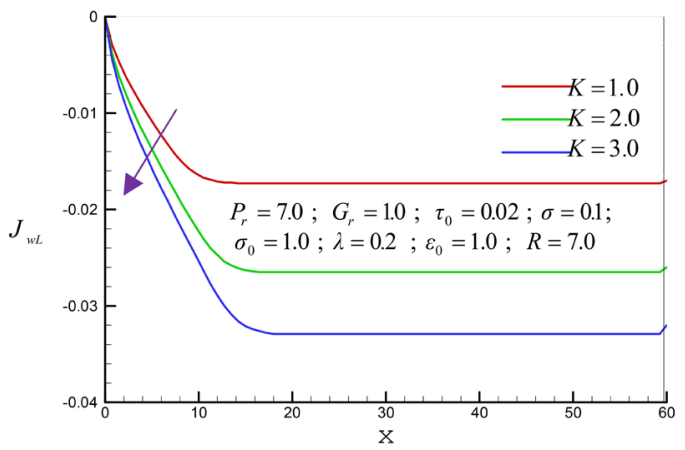

(a) 


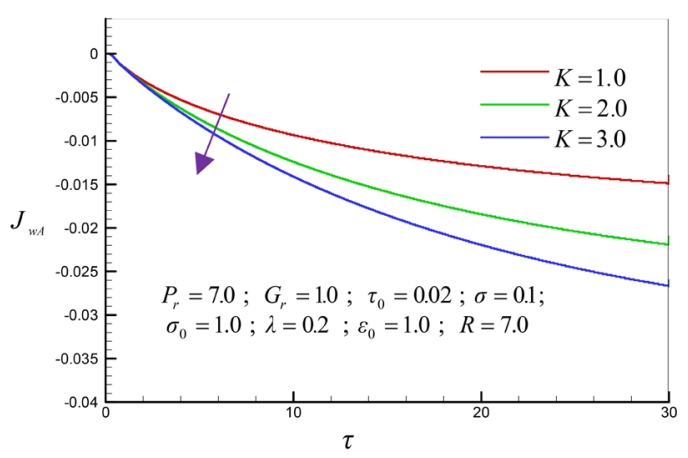

(b)

Figure 24. (a) Illustration of Current density at the wall $J_{w L}$ for different values of $K$; (b). Illustration of average Current density $J_{w A}$ for different values of $K$.

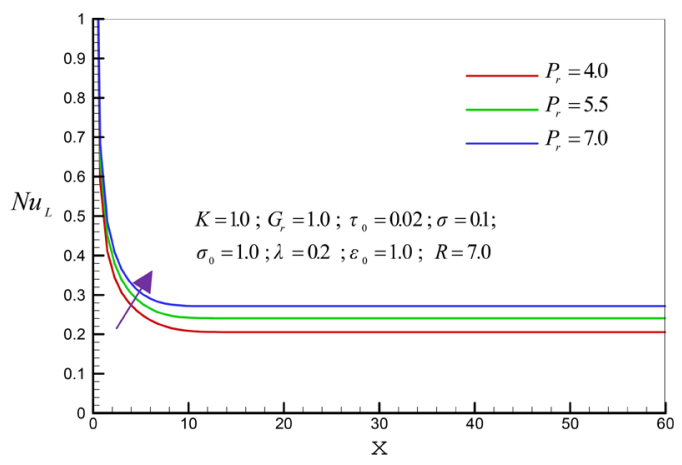

(a)

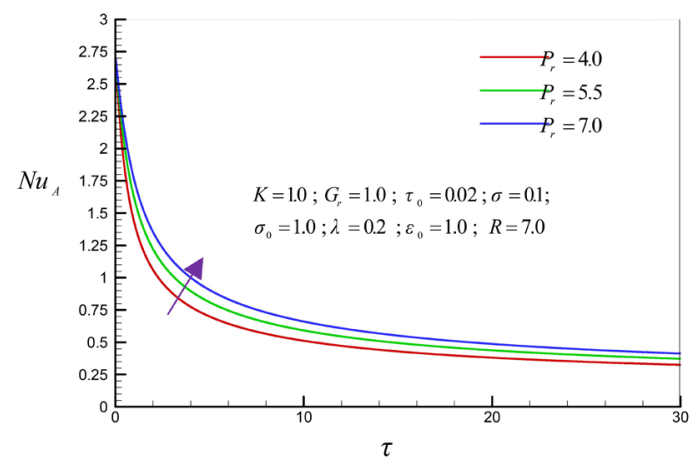

(b)

Figure 25. (a) Illustration of Nusselt number $N u_{L}$ for different values of $P_{r}$; (b): Illustration of average Nusselt Number $N u_{A}$ for different values of $P_{r}$

\subsection{Time and Mesh Sensitivity Test}

To get the steady-state solution, the computations are carried out for different time $t=20,25,29$ and 30 with time increment $\Delta t=0.001$ for the velocity distribution, which have shown in Figure 2. It is found that after $t=30$, there are very negligible changes. On the other hand, to choose the appropriate mesh, a solutions find out for different pairs of meshes like as $(m, n)=(70,70)$; $(m, n)=(80,80)$ and $(m, n)=(90,90)$ on the velocity distributions, which have shown in Figure 3. For those three chosen different values of meshes, the profiles 
are likely unchanged. There are same situations for the other distributions. Therefore our estimated steady-state time is at $t=30$ with time increment $\Delta t=0.001$ and mesh pair is $(m, n)=(80,80)$ with the fixed values of $G_{r}=1.0$; $P_{r}=7.0 ; R=7.0 ; \tau_{0}=0.02 ; \quad \sigma=0.1 ; \sigma_{0}=1.0 ; \lambda=0.2 ; \varepsilon_{0}=1.0 ; \quad K=1.0$ and $b=0.2$.

\subsection{Comparison with Previous Results}

Zakaria [20] investigated the influence of the Grashof number on the velocity $u$, which is shown in Figure 4(a). Here the velocity decreases with the increase of Grashof number $G_{r}$ But in Figure 4(b), it is found that the velocity increases with the same increasing values of Grashof number. In this case the maximum time has taken $t=1$.

\subsection{Primary Velocity Distributions}

Figure 5 depicts that the velocity $u$ is increased with the increase of $K$. In Figure 6, it is observed that the velocity $u$ is decreased with the increasing values of $P_{r}$. But in Figure 7, showed a cross-flow for the velocity, here velocity distribution is decreased within the interval $0<y<6$ (approx.) and thereafter it has very minor increasing effect with the increase of $R$. Figure 8 represented that the velocity has an increasing effect with the increase of $t_{0}$.

\subsection{Induced Magnetic Field Distributions}

Figure 9 and Figure 10 illustrate that the induced magnetic field distribution $H$ has a cross-flow for the different values of $G_{r^{r}}$ It is obvious that near the plate, $H$ has a minor increasing effect and thereafter found a large decreasing effect for increasing values of $G_{r}$ and $K$. Figure 11 represents that $H$ has a very minor increasing effect near the plate and thereafter a decreasing effect with the increase of $P_{r}$ But from Figure 12, it is observed that $H$ has an increasing effect with the rising values of $R$.

\subsection{Induced Electric Field Distributions}

Profiles in Figure 13 and Figure 14, represented that the induced electric field $E$ is decreased with the increase of $G_{r}$ and $K$ respectively. But $E$ has an increasing effect with the rising values of $P_{r}$ which is shown in Figure 15.

\subsection{Microrotation Distributions}

The microrotation $N$ has a cross-flow depicts in Figure 16. It has a decreasing effect within $0<y<2.9$ (approx.) and thereafter it has an increasing effect with the increase of $K$. But Figure 17 noticed that the increasing values of $R$, the micropolar rotation $N$ has a decreasing effect within $0<y<2.2$ (approx.) and then it has an increasing effect.

\subsection{Temperature Distributions}

Figure 18 displays the effect of the Prandtl number $P_{r}$ on the temperature $(\theta)$. 
As shown, temperature is decreasing with the increasing of $P_{r}$

\subsection{Skin-Friction, Current Density and Rate of Heat Transfer}

The effects of various parameters on local and average shear stress from the velocity profile have been investigated. The non-dimensional form of the local shear stress and average shear stress in $x$-direction is given by the relations $\tau_{L}=\left.\mu \frac{\partial u}{\partial y}\right|_{y=0}$ and $\tau_{A}=\left.\frac{1}{L} \int_{0}^{L} \mu \frac{\partial u}{\partial y}\right|_{y=0} \mathrm{~d} x$ respectively. From the temperature profile, the effects of various parameters on local and average Nusselt numbers have been calculated. The local Nusselt number and the average Nusselt number are given by $N u_{L}=-\left.\mu \frac{\partial \theta}{\partial y}\right|_{y=0}$ and $N u_{A}=-\left.\frac{1}{L} \int_{0}^{L} \mu \frac{\partial \theta}{\partial y}\right|_{y=0} \mathrm{~d} x$ respectively. Similarly, analyze the effects of various parameters on the local and average Sherwood numbers from the concentration field. The rate of mass transfer at the plate is defined as the Sherwood number; the local Sherwood number and the average Sherwood number is defined by $S h_{L}=-\left.\mu \frac{\partial \varphi}{\partial y}\right|_{y=0}$ and $S h_{A}=-\left.\frac{1}{L} \int_{0}^{L} \mu \frac{\partial \varphi}{\partial y}\right|_{y=0} \mathrm{~d} x$ respectively.

Figures (a) and (b) of Figures 19-22 are displayed the variations of the local shear stress and average shear stress respectively. It is obtained from Figure 19 and Figure 20 that the local (or average) shear stress increases with the increase of $G_{r}$ and $K$. The same effects of velocity are also represented. But from Figure 21 and Figure 22, show that both of the local and average shear stress decreases with the increase of $P_{r}$ and $R$ respectively.

Again, figures (a) and (b) of Figure 23, Figure 24 showed the variations of the local and average current density respectively. It is presented that $J_{w L}$ (or $J_{w}$ ) has both decreasing effect with the increasing value of $G_{r}$ and $K$. But Figure 25(a), Figure 25(b) have shown the variation of local (or average) Nusselt number for different values of the Prandtl number $\left(P_{r}\right)$, it depicts from the figure that Nusselt number increases with the increase of $P_{r}$.

\section{Conclusions}

In the present study, the influence of various values of Prandtl number, Grashof number, permeability parameter, micropolar parameter, electric conductivity, electric permeability and thermal relaxation time has been investigated. The non-linear coupled governing equations have been solved numerically and the main findings can be summarized as follows:

1) The velocity $u$ increases with the increase of $G_{r}, K$ and $\tau_{0}$, while it decreases with the increase of $P_{r}$ and $R$.

2) Induced magnetic field $H$ has cross-flow near the plate. But in major space, it has been increasing effect with the increase of $P_{r}$ and $R$, while it decreases with the increase of $G_{r}$ and $K$. 
3) Induced electric field $E$ increases with the increase of $P_{r}$, while it decreases with the increase of $G_{r}$ and $K$.

4) Microrotation $N$ has cross-flow for all the different values of all the parameters. First portion near the plate $N$ has increasing effect with the increase of $R$. Thereafter, it has a decreasing effect. But for $G_{r}$ and $K$, it has reverse effect.

5) Temperature $\theta$ decreases with the increase of $P_{r}$.

6) Local (or average) Shear stress increases of $G_{r}$ and $K$, while it decreases with the increase of $P_{r}$ and $R$.

7) Local (or average) Current density decreases with the increase of $G_{r}$ and $K$.

8) Local (or average) Nusselt number increases with the increase $P_{r}$

The accuracy of this work is qualitatively good in case of all the flow parameters.

\section{Conflicts of Interest}

The authors declare no conflicts of interest regarding the publication of this paper.

\section{References}

[1] Eringen, A.C. (1966) Theory of Micropolar Fluids. Journal of Mathematics and Mechanics, 16, 1-18. https://doi.org/10.1512/iumj.1967.16.16001

[2] Eringen, A.C. (1972) Theory of Thermo Micropolar Fluids. Journal of Mathematical Analysis and Applications, 38, 480-496. https://doi.org/10.1016/0022-247X(72)90106-0

[3] Ali, M.M., Chen, T.S. and Armaly, B.F. (1984) Natural Convection Radiation Interaction in Boundary Layer Flow over Horizontal Surface. AIAA Journal, 22, 1797-1803. https://doi.org/10.2514/3.8854

[4] Harutha, A. and Devasena, Y. (2016) MHD Mixed Convection Flow of a Micropolar Fluids through Porous Medium towards a Stagnation, Point on a Vertical Porous Surface. IOSR Journal of Mathematics, 12, 32-37. https://doi.org/10.9790/5728-1204043237

[5] Hudimoto, B. and Tokuoka, T. (1969) Two Dimensional Shears Flows of Linear Micro Polar Fluids. International Journal of Engineering Science, 7, 515-522. https://doi.org/10.1016/0020-7225(69)90036-6

[6] Rees, D.A.S. and Pop, I. (1998) Free Convection Boundary Layer Flow of a Micropolar Fluid from a Vertical Flat Plate. IMA Journal of Applied Mathematics, 61, 179-197. https://doi.org/10.1093/imamat/61.2.179

[7] Elbarbary, E.M.E. (2005) Chebyshev Finite Difference Method for the Solution of Boundary Layer. Applied Mathematics and Computation, 160, 487-498. https://doi.org/10.1016/j.amc.2003.11.016

[8] Nandhini, E. and Ramya, M. (2018) MHD Free Convection Flow in a Micropolar Fluid past an Inclined Stretching Sheet with Considering Viscous Dissipation and Radiation. International Journal of Scientific Research in Science, Engineering and Technology, 4, Issue1.

[9] Hassanien, I.A. and Glora, R.S.R. (1990) Heat Transfer to a Micropolar Fluid from a Non-Isothermal Stretching Sheet with Suction and Blowing. Acta Mechanica, 84, 191-199. https://doi.org/10.1007/BF01176097 
[10] Ahmad, K., Ishak, A. and Nazar, R. (2013) Micropolar Fluid Flow and Heat Transfer over a Nonlinearly Stretching Plate with Viscous Dissipation. Mathematical Problems in Engineering, 2013, Article ID: 257161. https://doi.org/10.1155/2013/257161

[11] Khonsari, M.M. and Brewe, D. (1989) On the Performance of Finite Journal Bearings Lubricated with Micro Polar Fluids. Tribiology Transactions, 32, 155-160. https://doi.org/10.1080/10402008908981874

[12] Ezzat, M.A. and Abd-Ellal, M.Z. (1997) Free Convection Effects on a Viscoelastic Boundary Layer Flow with One Relaxation Time through a Porous Medium. Journal of Franklin Institute, 334, 685-706. https://doi.org/10.1016/S0016-0032(96)00095-6

[13] Edlabe, N.T.M. and Mohammed, M.A.A. (2002) Heat and Mass Transfer in Hydrodynamic Flow of the Non-Newtonian Fluid with Heat Source over an Accelerating Surface through a Porous Medium. Chaos, Solitons and Fractals, 13, 907-917. https://doi.org/10.1016/S0960-0779(01)00066-2

[14] Edlabe, N.T.M. and Ouaf, M.E.M. (2006) Chebyshev Finite Difference Method for Heat and Mass Transfer in a Hydrodynamic Flow of a Micropolar Fluid past a Stretching Surface with Ohmic Heating and Viscous Dissipation. Applied Mathematics and Computation, 177, 561-571. https://doi.org/10.1016/j.amc.2005.07.071

[15] Aydin, O. and Pop, L. (2007) Natural Convection in a Differentially Heated Enclosure Filled with a Micropolar Fluid. International Journal of Thermal Science, 46, 963-969. https://doi.org/10.1016/j.ijthermalsci.2006.11.018

[16] Muthu, P., Rathish Kumar, B.V. and Chandra, P. (2008) A Steady of Micropolar Fluid in an Annular Tube with Application to Blood Flow. Journal of Mechanics in Medicine and Biology, 8, 561-576. https://doi.org/10.1142/S0219519408002541

[17] Glora, R.S.R. (1995) Unsteady Mixed Convection in Micropolar Boundary Layer Flow on a Vertical Plate. Fluid Dynamics Research, 15, 237-250. https://doi.org/10.1016/0169-5983(95)94957-U

[18] Hsu, T.H. and Wang, S.G. (2000) Mixed Convection of Micropolar Fluids in a Cavity. International Journal of Heat and Mass Transfer, 43, 1563-1572. https://doi.org/10.1016/S0017-9310(99)00242-2

[19] Lok, Y.Y., Amin, N. and Pop, I. (2006) Unsteady Mixed Convection Flow of a Micropolar Fluid near the Stagnation Point on a Vertical Surface. International Journal of Thermal Science, 45, 1149-1157. https://doi.org/10.1016/j.ijthermalsci.2006.01.015

[20] Zakaria, M. (2004) Problem in Electromagnetic Free Convection Flow of a Micropolar Fluid with Relaxation Time through a Porous Medium. Applied Mathematics and Computation, 151, 601-613. https://doi.org/10.1016/S0096-3003(03)00365-5 


\section{Nomenclature}

\begin{tabular}{|c|c|c|c|}
\hline$u^{+}$ & : Velocity components in $x^{+}$-direction & $\alpha$ & : Alfven velocity \\
\hline$v^{+}$ & : Velocity components in $y^{+}$- direction & $g$ & : Acceleration due to the gravity \\
\hline$N^{+}$ & : Dimensional Microrotation & $\mu_{0}$ & : Magnetic permeability \\
\hline$E^{+}$ & : Dimensional Induced electric field & $t^{+}$ & Dimensional time \\
\hline $\begin{array}{l}L \\
H^{+}\end{array}$ & : Magnetic induction along $x^{+}$- axis & & : Dimensionless time \\
\hline$H$ & : Constant magnetic induction along $y^{+}$- axis & & : Time increment \\
\hline$H_{0}$ & : Thermal relaxation time & $\begin{array}{l}\Delta t \\
\tau\end{array}$ & : Maximum time \\
\hline$\tau_{0}^{+}$ & : Electric permeability & & : Density of the fluid \\
\hline$\varepsilon_{0}^{+}$ & : Volumetric coefficient of thermal expansion & & : Magnetic diffusivity \\
\hline$\beta$ & : Temperature of the fluid & $U_{m}$ & : Microinertia density \\
\hline$T^{+}$ & : Constant temperature near the plate & & : Electric conductivity \\
\hline$T_{w}^{+}$ & : Temperature outside of the boundary layer & $\sigma^{\prime}$ & : Spin-gradient viscosity \\
\hline & : Electric conductivity & & : Grashof number \\
\hline$T_{\infty}$ & : Permeability of the porous medium & $G_{r}$ & : Prandtl number \\
\hline$\sigma_{0}^{+}$ & : Thermal conductivity & $P_{r}$ & : Microrotation parameter \\
\hline$\kappa$ & : Specific heat at the constant pressure & $R$ & : Permeability parameter \\
\hline$k$ & : Absolute viscosity & $K$ & : Dimensionless Material parameter \\
\hline$c_{p}$ & : Vortex viscosity & $\sigma$ & : Dimensionless Material parameter \\
\hline$\mu$ & : Dimensionless velocity component in $x$-direction & $b$ & : Dimensionless Material parameter \\
\hline$\mu^{*}$ & : Dimensionless velocity component in $y$-direction & $\lambda$ & : Dimensionless Material parameter \\
\hline$u$ & : Dimensionless microrotation & $\varepsilon_{1}$ & : Local shear stress \\
\hline V & : Dimensionless induced electric field & & : Average shear stress \\
\hline$N$ & : Dimensionless magnetic induction in $x$-direction & & : Local Nusselt number \\
\hline E & : Dimensionless temperature & $\tau_{x A}$ & : Average Nusselt number \\
\hline$H$ & & $N u_{L}$ & : Local Current density \\
\hline$\theta$ & & $N u_{A}$ & : Average Current density \\
\hline & & $J_{w L}$ & \\
\hline & & $J_{w A}$ & \\
\hline
\end{tabular}

